\title{
Risk-Adapted, Combined-Modality Therapy for Unfavorable Pediatric Hodgkin Lymphom
}

\author{
Amany M. Ali1, Amira M. Mohamed1, Shimaa Ahmed2, Mohamed Abdallah³, Tarek M. Alsaba", \\ Samar Mansour ${ }^{5}$
}

${ }^{1}$ Pediatric Oncology Department, South Egypt Cancer Institute, Assiut University, Assiut, Egypt

${ }^{2}$ Radiotherapy Department, South Egypt Cancer Institute, Assiut University, Assiut, Egypt

${ }^{3}$ Radiology Department, South Egypt Cancer Institute, Assiut University, Assiut, Egypt

${ }^{4}$ Pathology Department, South Egypt Cancer Institute, Assiut University, Assiut, Egypt

${ }^{5}$ Clinical Pathology Department, South Egypt Cancer Institute, Assiut University, Assiut, Egypt

Email: *shimaayoussif04@gmail.comh

How to cite this paper: Ali, A.M., Mohamed, A.M., Ahmed, S., Abdallah, M., Alsaba, T.M. and Mansour, S. (2018) RiskAdapted, Combined-Modality Therapy for Unfavorable Pediatric Hodgkin Lymphom. Journal of Cancer Therapy, 9, 545-558. https://doi.org/10.4236/jct.2018.97046

Received: May 24, 2018

Accepted: July 7, 2018

Published: July 10, 2018

Copyright $\odot 2018$ by authors and Scientific Research Publishing Inc. This work is licensed under the Creative Commons Attribution International License (CC BY 4.0).

http://creativecommons.org/licenses/by/4.0/

(c) (i) Open Access

\begin{abstract}
Background and Objectives: Risk-adapted therapy for children with HL is directed toward high survival, minimal toxicity and optimal quality of life, with long term follow up. We assess the impact of prognostic factors associated with local treatment failure of pediatric HL patients with unfavorable criteria treated with combined modality: Alternating ABVD (Doxorubicin, Bleomycin, Vinblastine and Decarbazine) and COEP (Cyclophosphamide, Oncovin, Etoposide and Prednisone) chemotherapy and response-based, involved-field radiation for newly diagnosed unfavorable pediatric HL patients, also will detect toxicities and long-term complications observed in the patients. Methods: This prospective study was carried out from January 2010 to January 2018, with a median follow up of 74 months (range 8 - 103 months). 54 patients were eligible for this study stratified into two groups: intermediate risk (IR) and high-risk group (HR). Patients were treated with (4 - 6 cycles) and (6 - 8 cycles) respectively of alternating ABVD/COEP chemotherapy followed by involved-field radiation therapy (IFRT): $15 \mathrm{~Gy}$ for patients achieved complete response, and $25.5 \mathrm{~Gy}$ for those achieved a partial response. Results: 27 patients were IR and 27 patients were HR. There were 16 treatment failures; 5 patients had progressive disease; and 11 patients had a relapse. 9 patients died from their disease progression. The 5-year overall survival (OS) and event-free survival (EFS) rates $( \pm \mathrm{SE})$ were $81.8 \% \pm 5.7 \%$ and $71.8 \% \pm$ $6.2 \%$ respectively. Multivariate analysis revealed that the only independent factor for inferior OS was radiotherapy. Conclusion: Treatment results of unfavourable HL patients in our study are satisfactory for with IR group but not for HR group who needs intensification of therapy. Radiotherapy is considered as a cornerstone in the treatment of the patients with unfavourable
\end{abstract}


criteria with better assessment of early responders needed by PET-CT to identify patients at risk for relapse.

\section{Keywords}

Risk-Adapted, Combined-Modality, Unfavourable Pediatric Hodgkin Lymphoma

\section{Introduction}

Hodgkin's lymphoma (HL) is a highly curable malignant disease. All children with newly diagnosed HL should be treated with curative intent. Good treatment planning with evaluation of site and extent of disease is an essential component of patient management [1]. With increasing understanding of the unique pathology of HL and the identification of novel targeted agents, there is an opportunity to further refine the therapy in order to improve the therapeutic index to maximize cure and reduce late toxicities of therapy [2].

Today the standard of care for the very large majority of children and adolescents with HL is risk and response-adapted, combined-modality therapy using low-dose, and involved-field radiation in conjunction with multiagent chemotherapy [1] [3]. Risk-adapted treatment protocols assign intensity of therapy according to risk features at diagnosis or response to initial therapy [4]. Although risk classifications vary across individual studies, the favourable or low risk is usually defined as localized (stage I/II) nodal involvement in the absence of B symptoms and nodal bulk. Unfavorable or high-risk presentations typically include the presence of B symptoms, bulky lymphadenopathy, hilar lymphadenopathy, involvement of four or more nodal regions, extranodal extension to contiguous structures, or advanced stage (III/IV). This approach has yielded excellent results, with long-term disease-free survival (DFS) of $85 \%$ to $100 \%$ in patients with early-stage disease and more than $60 \%$ in those with more advanced disease [5] [6].

Treatment outcomes have been correlated with many clinical factors, including male sex, nodular sclerosing histologyand grade, advanced stage, and the presence of B symptoms, anemia, leukocytosis, and bulky mediastinal disease. Some groups have proposed prognosticindices based on combinations of these and other laboratory parameters. In contrast to many other pediatric malignancies, reliable biologic prognostic factors have not been identified to guide therapeutic intensity for pediatric HL [7] [8].

Here, with long term follow up we will assess the impact of prognostic factors associated with local treatment failure of pediatric HL patients with unfavorable criteria treated with combined modality approach using alternating cycles of ABVD (Doxorubicin, Bleomycin, Vinblastine and Decarbazine) and COEP (Cyclophosphamide, Oncovin, Etoposide and Prednisone) chemotherapy and response-based, involved-field radiation therapy for newly diagnosed unfavorable pediatric HL patients, also will detect toxicities and long-term complications 
observed in the patients.

\section{Patients and Methods}

This prospective study was carried out at the Pediatric Oncology Department and Radiology Department, at South Egypt Cancer Institute (SECI) and Sohag Cancer Center from January 2010 to January 2018, the study was approved by our institutional review board and written informed consent was obtained from patient's family. Eligible patients had previously untreated pathologically proven HL, aged $\leq 18$ years. The patients were categorized as:

- Intermediate-risk (IR) group: includes early stage I/II with unfavorable criteria as B symptoms (unexplained loss of more than $10 \%$ of body weight in the preceding 6 months, presence of unexplained recurrent fever greater than $38^{\circ} \mathrm{C}$ in the preceding month, or and recurrent drenching night sweats in the preceding month), bulky disease which defined as extra thoracic nodal aggregate with adiameter greater than $6 \mathrm{~cm}$ [9] or mediastinal mass with a diameter more than one-third of the intrathoracic diameter measured at the level of T5 on an upright chest radiograph, an involvement of 4 or more lymph node sites, and extra nodal involvement and stage IIIA.

- High-risk (HR) group: includes stage IIIB and stage IV.

Exclusion criteria: We excluded patients with favorable criteria or received any treatment before.

\subsection{Pretreatment Evaluation}

For each patient, pretreatment evaluation was done by history and physical examination, complete laboratory investigations (complete blood picture (CBC), liver and kidney function, erythrocytic sedimentation rate (ESR), thyroid function tests, sex hormones), bilateral bone marrow biopsy, chest X-ray and Computed tomography (CT) neck, chest, abdomen and pelvis, echocardiograph, and pulmonary function test. Excisional biopsy was obtained from presenting lymphadenopathy; histopathological classification was done according to Revised European American Lymphoma classification/World Health Organization (REAL/WHO) classification system [10]. Patients were staged by Cotswold's' modification of Ann Arbor staging system [11].

\subsection{Treatment Plan}

All patients will receive combination chemotherapy of ABVD cycle alternating with COEP cycle (Table 1); patients will receive a different number of cycles according to risk group (4- 6 cycles for IR group and 6 - 8 cycles for HR group), cycle given every 28 days. The evaluation was done every 2 cycles. Patients achieved complete responses (CR) or partial response (PR) were received IFRT.

\subsection{Radiotherapy Technique}

Radiotherapy delivered in the supine position, CT Simulation with $3 \mathrm{~mm}$ cut thickness using intravenous contrast. Target volume we treat involved field 
Table 1. Chemotherapy protocols.

\begin{tabular}{ccc}
\hline Drug & Dosel Route & Day $(s)$ \\
\hline ABVD Protocol: & & \\
\hline Doxorubicin & $25 \mathrm{mg} / \mathrm{m}^{2} \mathrm{IV}$ & $\mathrm{d} 1,15$ \\
Bleomycin & $10 \mathrm{mg} / \mathrm{m}^{2} \mathrm{IV}$ & $\mathrm{d} 1,15$ \\
Vinblastin & $6 \mathrm{mg} / \mathrm{m}^{2} \mathrm{IV}$ & $\mathrm{d} 1,15$ \\
Decarbazine & $375 \mathrm{mg} / \mathrm{m}^{2} \mathrm{IV}$ & $\mathrm{d} 1,15$ \\
(repeat every28 days) & & \\
COEP Protocol: & & $\mathrm{d} 1,15$ \\
Cyclophosphamide & $800 \mathrm{mg} / \mathrm{m}^{2} \mathrm{IV}$ & $\mathrm{d} 1,15$ \\
Vincristine (Oncovin) & $1.5 \mathrm{mg} / \mathrm{m}^{2} \mathrm{IV}$ & $\mathrm{d} 1,15$ \\
Etoposide & $60 \mathrm{mg} / \mathrm{m}^{2} \mathrm{IV}$ & $\mathrm{d} 1,15$ then gradual \\
Prednisone & $40 \mathrm{mg} / \mathrm{m}^{2} / \mathrm{day} \mathrm{PO}$ & withdrawal \\
(repeat every28 days) & & \\
\hline
\end{tabular}

${ }^{*}$ Procarbazine is substituted by etoposide in COEP regimen.

radiotherapy (IFRT) typically encompasses both the Kaplan nodal regions containing the pathologically enlarged lymph nodes at the time of diagnosis and in addition to the nodal echelons immediately superior and inferior to the primary site. GTV (gross target volume) include a nodal disease in case of residual (PR). CTV (clinical target volume) delineated using prechemotherapy tumor size with image fusion when applicable, for mediastinum and paraaortic nodes. The transverse diameter is post-chemotherapy $+1.5 \mathrm{~cm}$ which includes entire nodal station and account for subclinical disease with normal structure excluding PTV (planning target volume) margin add for setup uncertainties and for internal motion. We treat by 3-dimensional planning take in consideration ICRU recommendation (international commission on radiation units and measurements).

Organs at risk and dose constraints: patients treated with thoracic RT, mean heart doses < 15 Gy the mean bilateral lung dose is <12 Gy; dose: 15 Gy for complete remission and $25.5 \mathrm{~Gy}$ in cases of partial remission (PR). For children less than 5 years consider bilateral radiotherapy to avoid growth asymmetry.

\subsection{Evaluation of Response}

Patient's response was evaluated by physical examination, laboratory investigations and radiological examination (CT scan) according to the initial positive findings every 2 cycles of chemotherapy. It was done according to revised RECIST guidelines: Complete response (CR): was defined as disappearance of all tumor-related constitutional symptoms and disappearance of all measurable nodal disease, Partial response (PR): was defined as at least $30 \%$ of all measurable lesions with disappearance of all constitutional symptoms if initially present, Stable disease (SD): neither PR nor PD criteria met, and Progressive disease (PD): $20 \%$ increase; no $\mathrm{CR}, \mathrm{PR}$, or SD documented before increased disease, or new lesion(s) [12].

\subsection{After-Therapy Follow up}

After completion of treatment, patients were followed every one month in the 
first year, every 3 months for next 2 years, every 6 months in the 4th and 5th years the annually. Follow-up was done by physical examination, ESR, repetition of previously positive radiological examination according to initial presentation (CT scans was only done in the 1st year of follow up then patients followed by ultrasonography). Additional examinations were performed as clinically indicated in patients presenting with clinical signs or symptoms suggestive of relapse.

Assessment of treatment-related organ toxicity was done annually after completion of therapy by physical examination, measurement of thyroid function tests for patients treated with cervical and upper mediastinal irradiation, pulmonary function studies for patients treated with chest radiation, echocardiogram, ECG for all patients and sex hormones in adolescents.

\subsection{Statistical Study}

The study cut off limit was January 2018. Overall survival (OS) was calculated from the first day of chemotherapy to the date of the last follow up or death due to any cause. Event-free survival (EFS) was calculated from the first day of chemotherapy to an event (death due to any cause, progression after PR, relapse or a second malignancy) or to the date of the last date of follow up contact for patients who did not experience any event [13]. OS and EFS were estimated with Kaplan-Meier method [14] and differences were assessed by the log-rank test [15]. Cox-regression used to estimate multivariate analysis [16].

\section{Results}

From January 2010 to January 2018, 54 newly diagnosed patients with unfavourable HL were enrolled in this study. The patient's characteristics are listed in Table 2. Twenty one of the patients (27 patients) grouped as IR group. Twenty two patients (38.9\%) were stage II, 23 patients (42.6\%) were stage III and 10 patients (18.52\%) were stage IV. The majority of patients (74.1\%) had systemic "B" symptoms, and $59.3 \%$ has proved to have mixed cellularity pathological subtype. Early response (CR after 1st 2 cycles of chemotherapy) only was reported in 16 patients (29.6\%).

\subsection{Survival Analysis}

At a median follow up of 74 months (range 8 - 103 months), 5-years EFS was $71.8 \% \pm 6.2 \%$ and 5 -years OS were $81.8 \% \pm 5.7 \%$. (Figure 1 and Figure 2 ).

Different factors affecting OS and EFS are listed in Table 3. As shown in Table 3 the only factors affect 5 -years OS are disease bulk, extranodal involvement and receiving radiotherapy with the highly significant difference between patients who receive radiotherapy or not $(93.4 \% \pm 4.7 \%$ versus $30 \% \pm 14.5 \%$ respectively) ( $\mathrm{p}<0.0001)$. Regarding 5 -years EFS, the risk group and radiotherapy are only factors statistically significant, also disease bulk and the stages how difference but it isn't statistically significant. 


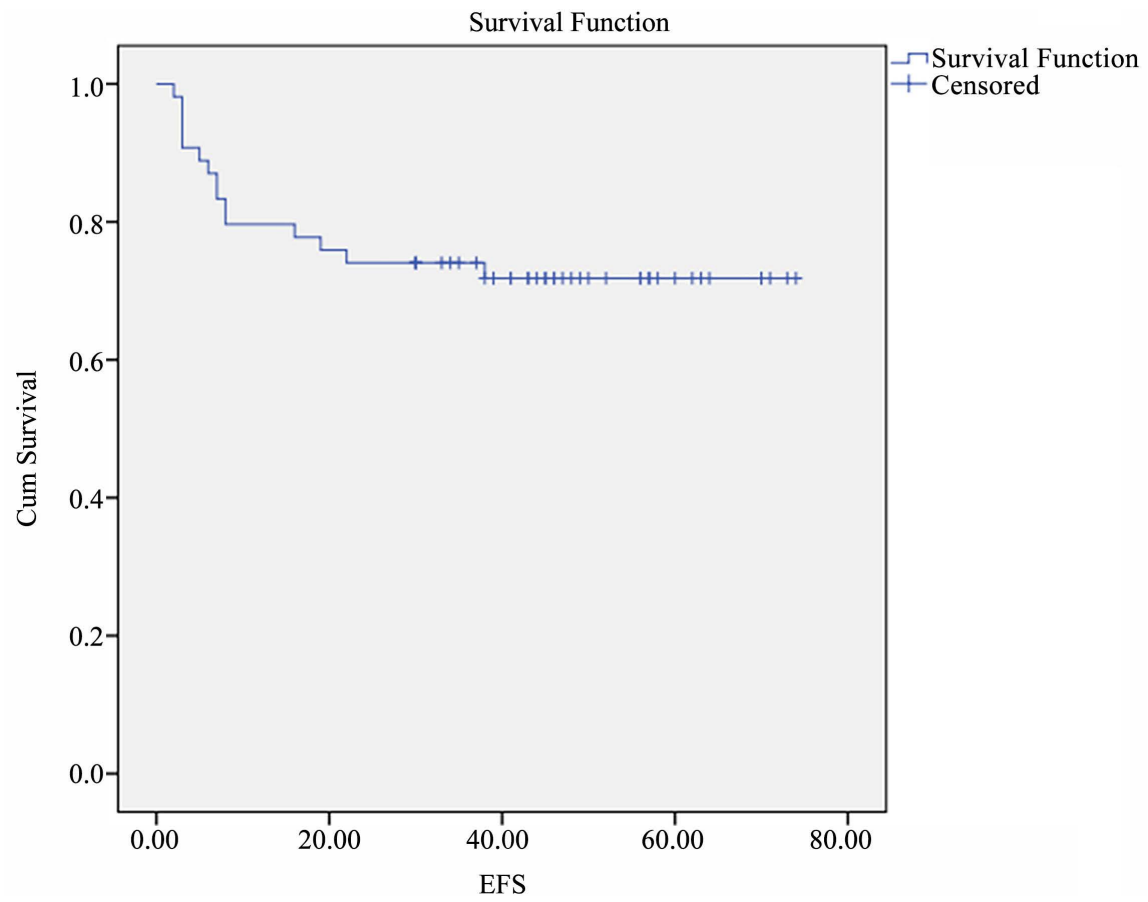

Figure 1. EFS of 54 patients is $71.8 \% \pm 6.2 \%$.

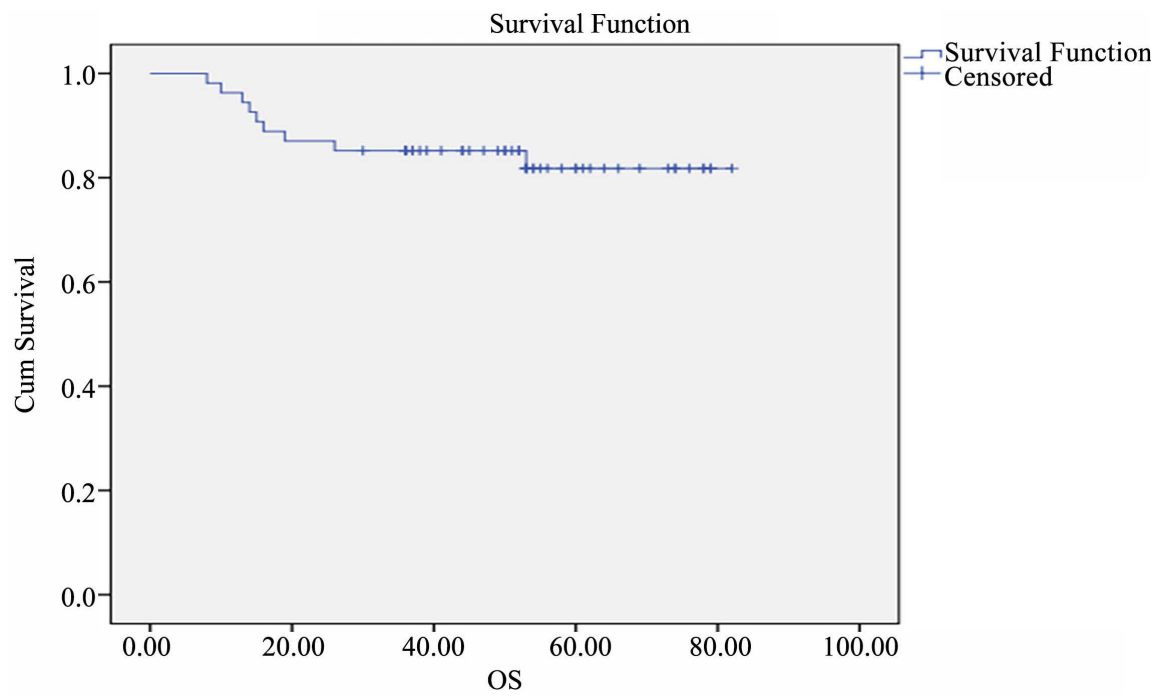

Figure 2. OS of 54 patients is $81.8 \% \pm 5.7 \%$.

Table 2. Clinical characteristics of 54 unfavorable HL patients.

\begin{tabular}{ccc}
\hline Patient Characteristics & No. of Patients & Percent (\%) \\
\hline Risk group & 27 & 50 \\
IR & 27 & 50 \\
HR & & \\
Age, years & $4-17$ & \\
Range & 8.9 & 68.5 \\
Median & 37 & 31.5 \\
$\leq 10$ & 17 & \\
$>10$ & & \\
\hline
\end{tabular}




\section{Continued}

\begin{tabular}{|c|c|c|}
\hline \multicolumn{3}{|l|}{ Gender } \\
\hline Male & 30 & \multirow{2}{*}{55.6} \\
\hline Female & 24 & \\
\hline Ratio & $1.3: 1$ & 44.4 \\
\hline \multicolumn{3}{|l|}{ Systemic symptoms } \\
\hline Absent $(\mathrm{A})$ & 14 & 25.9 \\
\hline Present (B) & 40 & 74.1 \\
\hline \multicolumn{3}{|l|}{ Stage } \\
\hline II & 21 & 38.9 \\
\hline III & 23 & 42.6 \\
\hline IV & 10 & 18.5 \\
\hline \multicolumn{3}{|l|}{ Bulky Disease } \\
\hline No & 31 & 57.4 \\
\hline Yes & 23 & 42.6 \\
\hline Mediastinal & 11 & 20.4 \\
\hline Abdominal & 5 & 9.3 \\
\hline Peripheral & 7 & 12.9 \\
\hline \multicolumn{3}{|l|}{ No. of Nodal Regions } \\
\hline$<4$ & 12 & 22.2 \\
\hline$\geq 4$ & 42 & 77.8 \\
\hline \multicolumn{3}{|l|}{ Extranodal } \\
\hline No & 34 & 62.9 \\
\hline Yes & 20 & 37.1 \\
\hline \multicolumn{3}{|l|}{ Histological subtypes } \\
\hline Mixed cellularity & 32 & \\
\hline Nodular sclerosis & 14 & $\begin{array}{l}59.3 \\
250\end{array}$ \\
\hline Lymphocytic rich & 5 & 25.9 \\
\hline Lymphocytic depletion & 3 & 9.3 \\
\hline \multicolumn{3}{|l|}{ Response } \\
\hline Early CR & 16 & 29.6 \\
\hline Late CR & 33 & 61.1 \\
\hline PD & 5 & 9.2 \\
\hline \multicolumn{3}{|l|}{ RTH } \\
\hline No & 10 & 18.4 \\
\hline yes & 44 & 81.4 \\
\hline
\end{tabular}

Table 3. Factors affecting OS and EFS.

\begin{tabular}{|c|c|c|c|c|}
\hline Variable & 5-Year OS (\%) & P-Value & 5-Year EFS (\%) & p-Value \\
\hline \multicolumn{5}{|l|}{ Risk group } \\
\hline IR & $88.9 \pm 6$ & \multirow{2}{*}{0.28} & $84.2 \pm 7.1$ & \multirow{2}{*}{0.026} \\
\hline HR & $72.4 \pm 10.8$ & & $59.3 \pm 9.3$ & \\
\hline \multicolumn{5}{|l|}{ Sex } \\
\hline Male & $74.7 \pm 8.5$ & \multirow{2}{*}{0.989} & $62.7 \pm 8.9$ & \multirow{2}{*}{0.145} \\
\hline Female & $91.7 \pm 5.4$ & & $83.3 \pm 7.6$ & \\
\hline \multicolumn{5}{|l|}{ Age } \\
\hline$\leq 10$ & $78.4 \pm 7.4$ & \multirow{2}{*}{0.497} & $75.7 \pm 7.1$ & \multirow{2}{*}{0.321} \\
\hline$>10$ & $88.2 \pm 8.1$ & & $63.5 \pm 4.5$ & \\
\hline
\end{tabular}




\section{Continued}

\begin{tabular}{|c|c|c|c|c|}
\hline Stage & & & & \multirow{4}{*}{0.108} \\
\hline II & $85.7 \pm 7.6$ & \multirow{3}{*}{0.46} & $80 \pm 9$ & \\
\hline III & $87 \pm 7$ & & $73.9 \pm 9.2$ & \\
\hline IV & $53.3 \pm 23.3$ & & $50 \pm 11.2$ & \\
\hline \multicolumn{5}{|l|}{ Histopathology } \\
\hline Mixed cellularity & $76.7 \pm 7.9$ & \multirow{4}{*}{0.568} & $65.1 \pm 8.5$ & \multirow{4}{*}{0.5} \\
\hline Nodular Scleroses & $85.7 \pm 9.4$ & & $78.6 \pm 11$ & \\
\hline Lymphocytic rich & 100 & & 100 & \\
\hline Lymphocytic Depletion & 100 & & $66.7 \pm 27.2$ & \\
\hline \multicolumn{5}{|l|}{ Extra nodal Involvement } \\
\hline Absent & $91.2 \pm 4.9$ & \multirow{2}{*}{0.036} & $78.8 \pm 7.2$ & \multirow{2}{*}{0.11} \\
\hline Present & $65.6 \pm 12.2$ & & $60 \pm 11$ & \\
\hline \multicolumn{5}{|l|}{ Systemic Symptoms } \\
\hline Absent $(\mathrm{A})$ & $92.9 \pm 6.9$ & \multirow{2}{*}{0.28} & $84.4 \pm 10.2$ & \multirow{2}{*}{0.2} \\
\hline Present (B) & $77.9 \pm 7.2$ & & $67.5 \pm 7.4$ & \\
\hline \multicolumn{5}{|l|}{ Bulky Disease } \\
\hline Absent & $93.5 \pm 4.4$ & \multirow{2}{*}{0.015} & $80 \pm 7.3$ & \multirow{2}{*}{0.09} \\
\hline Present & $61.6 \pm 13.6$ & & $60.9 \pm 10.2$ & \\
\hline \multicolumn{5}{|l|}{ Number of regions } \\
\hline$<4$ & $83.3 \pm 10.8$ & \multirow{2}{*}{0.963} & $83.3 \pm 710.8$ & \multirow{2}{*}{0.3} \\
\hline$\geq 4$ & $81.4 \pm 6.6$ & & $68.5 \pm 7.3$ & \\
\hline \multicolumn{5}{|l|}{ Early response } \\
\hline No & $80.4 \pm 7.4$ & \multirow{2}{*}{0.8} & $67.7 \pm 7.7$ & \multirow{2}{*}{0.36} \\
\hline Yes & $81.3 \pm 9.8$ & & $81.3 \pm 9.8$ & \\
\hline \multicolumn{5}{|l|}{ RT } \\
\hline No & $30 \pm 14.5$ & \multirow{2}{*}{$<0.0001$} & $30 \pm 14.5$ & \multirow{2}{*}{$<0.0001$} \\
\hline Yes & $93.4 \pm 4.7$ & & $80.8 \pm 6.1$ & \\
\hline
\end{tabular}

Multivariate analysis revealed that the only independent factor for inferior OS is radiotherapy ( $p=0.046$, HR: $2.6,95 \%$ CI: $0.08-0.41$ ).

\subsection{Treatment Failure}

There were $16(29.6 \%)$ treatment failures, 5 patients (9.2\%) had progressive disease (one patient was of IR group and 4 patients were HR). Eleven patients (20.4\%) relapsed ( 3 patients were IR and 8 patients were HR), seven patients relapsed outside the irradiated field; the other four relapsed within and outside the irradiated field. All failures were salvaged by second line chemotherapy ifosfamide, carboplatin, and etoposide (ICE protocol).

\subsection{Toxicity}

Treatment was well tolerated and without significant toxicity. All chemotherapy was given as outpatient therapy; they received full doses of chemotherapy with occasional delays due to neutropenia. Regarding the early complications, bone marrow suppression was the most frequent complication (15\%) especially in patients with advanced stage followed by repeated chest infection in five patients. Nausea and vomiting were observed in 7 patients (12.9\%) ranged from grade 1 - 2 . 
Acute hepatic toxicity occurred in 4 patients without delay of cycles. The acutegonadal failure occurred in a female patient (post-pubertal) after receiving pelvic radiotherapy in spite of oophoropexy; she received hormonal replacement therapy with recovery after 4 years with normalization of FSH level.

There were late effects in the form of subclinical hypothyroidism in two patients (3.7\%), asymptomatic left ventricular dysfunction in 3 patients $(5.5 \%)$ and restrictive pulmonary function in one patient $(1.8 \%)$. No cases of second malignancy or growth dissymmetry were recorded in our study.

\section{Discussion}

Risk-adapted regimens seek to maintain disease control while reducing therapy-related complications. Therefore, this approach may reduce therapy for patients with favourable diagnostic features or intensify therapy for patients with unfavourable disease presentations [1].

There are differences between the United States and European investigators in the assignment of risk in protocols. Investigators agree on low-risk patients with favourable features. These generally include patients with stage I and II disease, A status, and no bulk. Patients with stage I and II disease with bulk and selected other features including mixed cellularity histology are often classified as early-stage unfavourable patients in German Hodgkin Study Group (GHSG) and European protocols and as intermediate-risk patients in protocols generated in the National Clinical Trials Network (NCTN)/North America. Intermediate-risk patients include stage I and II patients with B symptoms and bulk as well as stage III and IV patients with A symptoms. Advanced-stage III and IV patients include those with bulk and B symptoms for groups in the United States/North America and Europe. There remains a challenge in overlap areas between groups and we will need to be careful in reviewing publications, understanding that there may be differences in clinical trial populations within specific groups [17].

Chemotherapy used for this group includes derivatives combinations of MOPP and ABVD. COPP has more recently replaced MOPP because cyclophosphamide is less myelosuppressive and leukemogenic than mechlorethamine. Etoposide is frequently added to enhance treatment response and reduce cumulative doses of alkylating and anthracycline chemotherapy [18].

There is a dual and conflicted approach for modern management of unfavourable early stage/intermediate risk HL and advanced stage HL between the United States and the GHSG are asymmetric as the US studies generally began treatment with $A B V D$ with attenuation or intensification drove by the response to treatment. The German group established the paradigm to initiate care in advanced-stage patients with more intensive therapy with dose/therapy adjustment to a less-intensive regimen if the patient achieved a good response to initial management. The GHSG investigators demonstrated improved CR and event-free survival rates with more intensive initial therapy and as a consequence established a strategy to limit first-course failure perhaps at the expense of toxicity. 
American and some European investigators suggest that because patients can be rescued with secondary/relapse therapy, achieving up-front full CR at the expense of toxicity remains less desirable [19] [20].

The role of RT has evolved over time, efforts at radiation dose/volume modification and therapeutic titration became embedded in clinical trials in both Europe and the United States/North America.

The European German Society for Paediatric Oncology and Haematology GPOH-HD 95 study showed that intermediate and high-risk patients had significantly better relapse-free survival when treated with RT following a complete response to chemotherapy compared with no additional treatment, which is the same with our results.

Early- and intermediate-risk patients with CR to chemotherapy may not require RT based on metabolic imaging. Protocols in advanced-stage patients evaluate the role of RT directed to areas at the presentation that do not fully respond to chemotherapy and/or sites of bulk disease at presentation (mediastinum) in COG trials [17].

In the current study, the median age was 8.9 years which matched with other studies [21] [22] and most of our patients (68.5\%) were under the age of 10 years which agrees with [23] and differ from [22] [24] [25]. There is a predominance of males in our study (1.3:1) like that reported by other studies [21] [24].

As regarding stage of the disease, patients with stage III were observed to be more common than stage II and IV in our patients which is similar to [23] and unlike which is reported by [22]. $74.1 \%$ of patients had systemic "B" symptoms which are more than reported in studies [22] [26]. Bulky disease was seen in 23 patients (42.6\% which is lower than reported by [22] [27]). Extranodal involvement occurred in 20 patients (37\% which in agreement to what was reported by [6] and less than reported by [28]).

Regarding risk stratification, we reported that half of the patients in our study stratified as HR group which unlike to [22] [29] [30] which can be explained by differences in risk assignment and number of patients.

Although nodular sclerosis histological subtype was reported to be the predominant histological subtype by [24] [28], mixed cellularity subtype was the predominant type $59.3 \%$ in our study which is matched with other studies [21] [23] [31]. This can explain the predominance of lower age $\leq 10$ years at diagnosis, advanced stage and " $\mathrm{B}$ " symptoms which is highly prevalent in developing countries rather than developed countries [27].

At a median follow up of 5-year EFS and OS were $71.8 \% \pm 6.2 \%$ and $81.8 \% \pm$ $5.2 \%$ respectively for all patients. The 5 -years EFS was $84.2 \% \pm 7.1 \%$ and $59.3 \%$ $\pm 9.3 \%$ for IR and HR groups which in concordance with Hudson et al., Gordon et al. and Friedman et al. [6] [24] [26] but is inferior to results regarding the HR group published by Schwartz et al. and Kelly et al. [22] [25]. The limited cumulative doses of alkylating agents and anthracycline chemotherapy in combination with low-dose, involved-field radiation seem to compromise disease control in this high-risk group. 
There is difference in 5-years EFS for patients with unfavorable early stage, stage III and IV $(80 \% \pm 9 \%, 73.9 \% \pm 9.3 \%$ and $50 \% \pm 11.2 \%$ respectively) but it isn't significant ( $\mathrm{p}=0.1$ ) which was like Hudson et al. [6] but it is unlike Droffel et al. and Schwartz et al. [22] [30].

Early response to chemotherapy seems to affect 5 years EFS in our patients $(81.3 \% \pm 9.8 \%$ for early responders and $67.7 \% \pm 7.7 \%$ for late responders $)$ but it was not translated to be significant $(\mathrm{P}=0.36)$, the difference is similar to which was reported by Friedman et al. [24]. The interim response in our study based on CT evaluation and recent current Euronet trials incorporates both CT and positron emission tomography (PET) after two cycles of chemotherapy for better assessment of patients not in CR assigned to radiation therapy [9].

In our study, there was significant difference between patients received or not received RT in 5-years EFS $80.8 \% \pm 6.1 \%$ versus $30 \% \pm 14.5 \%(\mathrm{p} \leq 0.0001)$ and 5 -years OS $93.4 \% \pm 4.7 \%$ versus $30 \% \pm 14.5 \%$ ( $\mathrm{p} \leq 0.0001$ ) similar to which reported by Dorffel et al. who also reported that omission of RT in patients with chemotherapy-induced CR proved to be safe only for patients with early-stage disease [30] [32].

\section{Conclusion}

Treatment results of unfavourable HL in the pediatric oncology department, at SECI treated with risk-adapted, response-based combined modality approaches are satisfactory to patients with IR group but not for HR group who needs intensification of therapy. Radiotherapy is considered a cornerstone in the treatment of the patients with unfavourable criteria with better assessment of early responders needed by PET-CT to identify patients at risk for relapse.

\section{Conflict of Interests}

The authors declare that they have no conflict of interests.

\section{References}

[1] Metzger, M., Krasin, M.J., Hudson, M.M., et al. (2011) Hodgkin Lymphoma. In: Philip, A., Pizzo, M.D. and Poplack, D.G. Eds., Principles and Practice of Pediatric Oncology, 6th Edition, Lippincott Williams and Wilkins, Philadelphia, 638-662.

[2] Kelly, K.M. (2015) Hodgkin Lymphoma in Children and Adolescents: Improving the Therapeutic Index. Blood, 126, 2452-2458.

https://doi.org/10.1182/blood-2015-07-641035

[3] Mc Dowell, H.P., Messahel, B. and Oberlin, O. (2006) Hodgkin's Disease. In: Imbach, P., Kühne, T. and Arceci, R.J., Eds., Pediatric Oncology, 3rd Edition, Springer, Berlin, 268-286.

[4] Donaldson, S.S., Hudson, M.M., Lamborn, K.R., et al. (2002) VAMP and Low-Dose, Involved-Field Radiation for Children and Adolescents with Favorable, Early-Stage Hodgkin's Disease: Results of a Prospective Clinical Trial. Journal of Clinical Oncology, 20, 3081-3087. https://doi.org/10.1200/JCO.2002.12.101

[5] Pacquement, H., Landman-Parker, J., Leblanc, T., et al. (2000) Localized Childhood Hodgkin's Disease: Response-Adapted Chemotherapy with Etoposide, Bleomycin, 
Vinblastine, and Prednisone before Low-Dose Radiation Therapy-Results of the French Society of Pediatric Oncology Study MDH90. Journal of Clinical Oncology, 18, 1500-1507. https://doi.org/10.1200/JCO.2000.18.7.1500

[6] Hundson, M.M., Krasin, M., Link, M.P., et al. (2004) Risk-Adapted, Combined-Modality Therapy with VAMP/COP and Response-Based, Involved-Field Radiation for Unfavorable Pediatric Hodgkin's Disease. Journal of Clinical Oncology, 22, 4541-4550. https://doi.org/10.1200/JCO.2004.02.139

[7] Schellong, G., Potter, R., Bramswig, J., et al. (1999) High Cure Rates and Reduced Long-Term Toxicity in Pediatric Hodgkin's Disease: The German-Austrian Multicenter Trial DAL-HD-90. Journal of Clinical Oncology, 17, 3736-3744. https://doi.org/10.1200/JCO.1999.17.12.3736

[8] Hutchinson, R.J., Fryer, C.J., Davis, P.C., et al. (1998) MOPP or Radiation in Addition to ABVD in the Treatment of Pathologically Staged Advanced Hodgkin's Disease in Children: Results of the Children's Cancer Group Phase III Trial. Journal of Clinical Oncology, 16, 897-906. https://doi.org/10.1200/JCO.1998.16.3.897

[9] Kelly, K.M. (2012) Management of Children with High-Risk Hodgkin Lymphoma. British Journal of Haematology, 157, 3-13. https://doi.org/10.1111/j.1365-2141.2011.08975.x

[10] Eberle, F.C., Mani, H. and Jaffe, E.S. (2009) Histopathology of Hodgkin's Lymphoma. The Cancer Journal, 15, 129-137. https://doi.org/10.1097/PPO.0b013e31819e31cf

[11] Lister, T.A., Crowther, D., Sutcliffe, S.B., et al. (1989) Report of a Committee Convened to Discuss the Evaluation and Staging of Patients with Hodgkin's Disease: Cotswolds Meeting. Journal of Clinical Oncology, 7, 1630-1636. https://doi.org/10.1200/JCO.1989.7.11.1630

[12] Eisenhauer, E.A., Therasse, P., Bogaerts, J., et al. (2009) New Response Evaluation Criteria in Solid Tumours: Revised RECIST Guideline (Version 1.1). European Journal of Cancer, 45, 228-247. https://doi.org/10.1016/j.ejca.2008.10.026

[13] Peto, R. and Peto, J. (1972) Asymptotically Efficient Rank Invariant Test Procedures. Journal of the Royal Statistical Society. Series A, 135, 185-198. https://doi.org/10.2307/2344317

[14] Kaplan, E.L. and Meier, P. (1958) Nonparametric Estimation from Incomplete Observations. Journal of the American Statistical Association, 53, 457-481. https://doi.org/10.1080/01621459.1958.10501452

[15] Savage, I. (1956) Contributions to the Theory of Rank Order Statistics: The Two Sample Case. The Annals of Mathematical Statistics, 27, 590-615. https://doi.org/10.1214/aoms/1177728170

[16] Cox, D.R. (1972) Regression Models and Life-Tables. Journal of the Royal Statistical Society: Series B, 42, 187-220.

[17] Fitzgerald, T.J. and Bishop-Jodoin, M. (2017) Hodgkin Lymphoma: Differences in Treatment between Europe and the United States/North America: Evolving Trends in Protocol Therapy. Clinical Medicine Insights. Oncology, 12, 1-12.

[18] Friedmann, A.M., Hudson, M.M., Weinstein, H.J., et al. (2002) Treatment of Unfavorable Childhood Hodgkins Disease with VEPA and Low-Dose, Involved-Field Radiation. Journal of Clinical Oncology, 20, 3088-3094.

https://doi.org/10.1200/JCO.2002.03.051

[19] Ballova, V., Ruffer, J.U., Haverkamp, H., et al. (2005) A Prospectively Randomized Trial Carried out by the German Hodgkin Study Group (GHSG) for Elderly Patients with Advanced Hodgkin's Disease Comparing BEACOPP Baseline and 
COPP-ABVD (Study HD9 Elderly). Annals of Oncology, 16, 124-131. https://doi.org/10.1093/annonc/mdi023

[20] Gallamini, A., Hutchings, M., Rigacci, L., et al. (2007) Early Interim 2-[18F]Fluoro2-Deoxy-D-Glucose Positron Emission Tomography Is Prognostically Superior to International Prognostic Score in Advanced Stage Hodgkin's Lymphoma: A Report from a Joint Italian-Danish Study. Journal of Clinical Oncology, 25, 3746-3752. https://doi.org/10.1200/JCO.2007.11.6525

[21] Ghazaly, M.H., Ali, A.M., Hamza, H.A. and Mohamed, A.M. (2014) Risk-Adapted Therapy of Pediatric Hodgkin Lymphoma in Upper Egypt.

[22] Schwartz, C.L., Constine, L.S., Villaluna, D., et al. (2009) A Risk-Adapted, Response-Based Approach Using ABVE-PC for Children and Adolescents with Intermediate- and High-Risk Hodgkin Lymphoma: The Results of P9425. Blood, 114, 2051-2059. https://doi.org/10.1182/blood-2008-10-184143

[23] Gao, Y.J., Tang, J.Y., Pan, C., et al. (2013) Risk-Adapted Chemotherapy without Procarbazine in Treatment of Children with Hodgkin Lymphoma. World Journal of Pediatrics, 9, 32-35. https://doi.org/10.1007/s12519-012-0390-0

[24] Friedman, D.L., Chen, L., Wolden, S., et al. (2014) Dose-Intensive Response-Based Chemotherapy and Radiation Therapy for Children and Adolescents with Newly Diagnosed Intermediate-Risk Hodgkin Lymphoma: A Report from the Children's Oncology Group Study AHOD0031. Journal of Clinical Oncology, 32, 3651-3658. https://doi.org/10.1200/JCO.2013.52.5410

[25] Kelly, K.M., Sposto, R., Hutchinson, R., et al. (2011) BEACOPP Chemotherapy Is Highly Effective Regimen in Children and Adolescents with High-Risk Hodgkin Lymphoma: A Report from the Children's Oncology Group. Blood Journal, 117, 2596-2603. https://doi.org/10.1182/blood-2010-05-285379

[26] Gordon, L.I., Hong, F., Fisher, R.I., et al. (2013) Randomized Phase III Trial of ABVD versus Stanford V with or without Radiation Therapy in Locally Extensive and Advanced-Stage Hodgkin Lymphoma: An Intergroup Study Coordinated by the Eastern Cooperative Oncology Group (E2496). Journal of Clinical Oncology, 31, 684-691. https://doi.org/10.1200/JCO.2012.43.4803

[27] Ebeid, E.N., Zaghloul, M. and Khairy, M. (2006) Clinical Outcome of Pediatric Hodgkin's Disease in Upper Egypt. Journal of Clinical Oncology, 24, 9035.

[28] Engert, A., Goergen, H., Markova, J., et al. (2017) Reduced-Intensity Chemotherapy in Patients with Advanced-Stage Hodgkin Lymphoma. Updated Results of the Open-Label, International, Randomized Phase 3 HD15 Trial by the German Hodgkin Study Group. HemaSphere, 1, e5. https://doi.org/10.1097/HS9.0000000000000005

[29] Mauz-Korholz, C., Hasenclever, D., Dorffel, W., et al. (2010) Procarbazine-Free OEPA-COPDAC Chemotherapy in Boysand Standard OPPA-COPP in Girls Have Comparable Effectiveness in Pediatric Hodgkin's Lymphoma: The GPOH-HD-2002 Study. Journal of Clinical Oncology, 28, 3680-3686.

https://doi.org/10.1200/JCO.2009.26.9381

[30] Dorffel, W., Luders, H., Ruhl, U., et al. (2013) Treatment of Children and Adolescents with Hodgkin Lymphoma without Radiotherapy for Patients in Complete Remission after Chemotherapy: Final Results of the Multicenter Trial GPOH-HD95. Journal of Clinical Oncology, 31, 1562-1568. https://doi.org/10.1200/JCO.2012.45.3266

[31] Ali, A., Sayed, H., Farrag, A. and El-Sayed, M. (2010) Risk-Based Combined-Modality Therapy of Pediatric Hodgkin's Lymphoma: A Retrospective Study. Leukemia Re- 
search, 34, 1447-1452. https://doi.org/10.1016/j.leukres.2010.06.012

[32] Wolden, S.L., Chen, L., Kelly, K.M., et al. (2012) Long-Term Results of CCG 5942: A Randomized Comparison of Chemotherapy with or without Radiotherapy for Children with Hodgkin's Lymphoma-A Report from the Children's Oncology Group. Journal of Clinical Oncology, 30, 3174-3180.

https://doi.org/10.1200/JCO.2011.41.1819 